\title{
Peri-procedural anticoagulation in patients undergoing ablation for atrial fibrillation
}

\author{
Sara R. Vazquez ${ }^{a,}{ }^{*}$, Stacy A. Johnson ${ }^{b}$, and Matthew T. Rondina ${ }^{c, d, e}$ \\ aUniversity of Utah Thrombosis Service, University of Utah, Department of Pharmacy Services, \\ 675 Arapeen Drive, Suite 100, Salt Lake City, UT 84108, United States \\ bDepartment of Internal Medicine, University of Utah School of Medicine, United States \\ 'Department of Internal Medicine, University of Utah Health Sciences Center, United States \\ dUniversity Thrombosis Service, University of Utah Health Sciences Center, United States \\ eTranslational Research, University of Utah Health Sciences Center, United States
}

\begin{abstract}
Radiofrequency catheter ablation is being used with increasing frequency as a strategy to manage atrial fibrillation. Patients undergoing this procedure are at increased short-term risk of thromboembolism for several days and up to 4 weeks or longer after their ablation, and anticoagulation management surrounding the ablation procedure remains controversial. Although no conclusive recommendations can be made, published guidelines and data support therapeutic anticoagulation with warfarin for 3 weeks prior and intravenous heparin during the ablation. Warfarin may either be continued through the ablation or stopped 2-5 days prior. If the latter approach is chosen, a pre-ablation bridging strategy of enoxaparin $1 \mathrm{mg} / \mathrm{kg}$ twice daily is reasonable in selected patients unless the patient's bleeding risk dictates using a lower dose regimen $(0.5 \mathrm{mg} / \mathrm{kg}$ twice daily) or avoiding bridging altogether. Fewer data are available for postablation management strategies, and current practice patterns are based largely on single-center experiences in smaller, non-randomized studies. For lower risk patients $\left(\mathrm{CHADS}_{2} 0-1\right)$, either warfarin or aspirin may be utilized without bridging. In higher thromboembolic risk patients $\left(\mathrm{CHADS}_{2} \geq 2\right)$, either enoxaparin ( $1 \mathrm{mg} / \mathrm{kg}$ twice daily) or heparin may be started within the first 12-24 h post-procedure. For patients with bleeding risk factors, enoxaparin may be subsequently reduced to $0.5 \mathrm{mg} / \mathrm{kg}$ until the INR is therapeutic, although the efficacy of this lower dosing regimen has not been well studied. In accordance with national guidelines, warfarin should be continued post-ablation for a minimum of 2 months and then indefinitely in patients with a $\mathrm{CHADS}_{2}$ score $\geq 2$.
\end{abstract}

\section{Keywords}

Atrial Fibrillation; Ablation; Anticoagulation; Low-molecular weight heparin; Unfractionated heparin; Warfarin; Peri-procedural; Bridging; Stroke; Transient Ischemic Attack; Thromboembolism

\footnotetext{
(C) 2009 Elsevier Ltd. All rights reserved.

*Corresponding author. Tel.: +1 801581 3449; fax: +1 801585 7978. sara.vazquez@hsc.utah.edu (S.R. Vazquez).

Conflict of interest statement

The authors have no conflicts of interest to disclose.
} 
Atrial fibrillation (AF) is one of the most common, sustained arrhythmias and is associated with significant morbidity and mortality. Although there is still controversy regarding the best management approach to $\mathrm{AF}$, most providers choose a strategy of either ventricular rate or rhythm control along with chronic anticoagulation to prevent thromboembolic (TE) complications [1]. More recently, in addition, or at times, as an alternative to these approaches, catheter-directed ablation is being used with increasing frequency in patients with symptomatic, refractory AF.

Regardless of what strategy is used, AF may result in TE - often from thrombus in the left atrium or atrial appendage - and accounts for nearly $15 \%$ of all strokes in the US [2], with an annual incidence of stroke of up to $6 \%$ or even higher in some patients [3]. The risk of stroke in AF is neither static nor uniform, may depend on both clinical and echocardiographic factors, and usually requires an individualized approach to assess stroke risk and determine appropriate AC management. Re-estimation of the estimated stroke risk is recommended as a patient's risk may change over time, and alternative therapy may be indicated.

Although recent meta-analysis data failed to identify one particular stroke risk assessment scoring system that is clearly superior to other validated systems[4], the $\mathrm{CHADS}_{2}$ score is commonly used to assess baseline stroke-risk and guide antithrombotic therapy (Table 1) [5]. Most of the published reports of peri-ablation anticoagulation reviewed in this paper utilized the $\mathrm{CHADS}_{2}$ scoring system and thus, we will also use this same risk assessment tool. In patients with a $\mathrm{CHADS}_{2}$ score of $\geq 2$, warfarin effectively reduces the risk of stroke and is recommended for chronic anticoagulation [1]. In AF patients with a $\mathrm{CHADS}_{2}$ score of $0-1$ - where the risk of stroke may be lower - warfarin is more controversial, given its narrow therapeutic window and risk of major or life-threatening bleeding, and either warfarin or aspirin are recommended for chronic anticoagulation, according to patient and provider preferences [6,7]. Although widely used, several limitations exist with the classical $\mathrm{CHADS}_{2}$ scoring system. Anticoagulant selection for the "intermediate risk" group (score 1-2), encompassing $>60 \%$ of patients, is determined by clinician preference [8] and fear of warfarin-related bleeding complications may result in suboptimal TE prophylaxis.

Additionally, the $\mathrm{CHADS}_{2}$ scoring neglects other known AF-related stroke risk factors (female sex, age 65-74, peripheral arterial disease), and lacks validation in the periprocedural period $[8,9]$.

AF ablation, if successful long-term, may reduce the risk of future stroke and transient ischemic attack (TIA). However, somewhat paradoxically, AF patients are at increased short-term risk of TE for several days, and up to four weeks, after their ablation [10]. This pro-thrombotic period may result in a higher, albeit transient, TE risk in AF patients who were previously judged to be low-risk. Although the precise mechanism(s) by which ablation induces this pro-thrombotic effect are not clearly understood, several factors are implicated. Transseptal sheath placement can precipitate thrombus formation on the catheter or sheath during the procedure (although catheter thrombus may be minimized with adequate peri-procedural anticoagulation and an open-irrigated tip) or in the left atrial appendage (LAA) [11-13]. Delivery of radiofrequency energy during the ablation disrupts the endocardium, possibly exposing procoagulant proteins (e.g. tissue factor) and activating the clotting cascade [14]. "Char" (hard coagulum) from tissue heating and denaturation and aggregation of plasma proteins [15] may form and systemically embolize [11]. Additionally, the atrial tissue may be stunned for several weeks, or even months, post-procedure leading to impairment of normal contraction [16].

Reported rates of peri-procedural TE complications are 0.5-2.8\% [11,17-19] and may be higher in patients with a $\mathrm{CHADS}_{2}$ score $\geq 2$, patients with a history of stroke [10], patients 
with persistent rather than paroxysmal AF [1], and patients with marked left atrial enlargement or a dilated LAA [20].

As the number of AF ablation procedures increases, especially in higher-risk and older patients, comprehensive and careful peri-procedural anticoagulation management based on best-available data and individualized for each patient is paramount. Although preprocedure warfarin (goal international normalized ratio [INR] 2-3) and intra-procedure intravenous unfractionated heparin (IV UFH) with a target activated clotting time (ACT) of $\geq 300$ seconds are routinely used, practice patterns for other peri-procedural anticoagulation (e.g. post-procedure heparinoids, antiplatelet agents, and duration of warfarin) vary widely. These differences are due, in part, to a paucity of adequately powered studies and, in the studies published to date, heterogeneity in patient populations, type of bridging strategy employed, and outcomes. These limitations underscore the need for caution when interpreting published data and, in the authors' opinion, the need for an individualized peri-procedural anticoagulation management plan for most patients. A more detailed list of the studies referred to in this review can be found in Appendix A.

\section{Should AF patients receive pre-procedural bridging therapy?}

Current guidelines recommend anticoagulating AF patients with warfarin (INR goal 2-3) for a minimum of 3 weeks prior to either electrical or chemical cardioversion [1,21]. Given the risk of peri-procedural TE events, expert consensus statements extend these recommendations to include ablation procedures [22]. For patients either refusing or who are intolerant to oral anticoagulation, antiplatelet agents, although less effective than warfarin in preventing TE, are a recommended alternative, especially in lower TE risk patients (i.e those with paroxysmal $\mathrm{AF}$ and/or a $\mathrm{CHADS}_{2}$ score of 0) [10,23-25].

Warfarinmay be stopped, at the clinician's preference, $2-5$ days prior to the procedure $[10,11,23,24,26-32]$. Alternatively, and as is done at our center and others, warfarin may be continued during the procedure without interruption. In two studies of lower risk patients (mean INR \pm standard deviation day of procedure $2.7 \pm 0.5$ and $2.8 \pm 0.7$ ), continuing warfarin during the procedure was not associated with an increased risk of peri-procedural bleeding $[27,33]$.

In patients where warfarin is stopped several days pre-ablation, either IV UFH or low molecular weight heparins (LMWH) may be used as "bridge" therapy (e.g. between the timewarfarin is discontinued and the procedure begins) [10,22-27,29,30,34-36]. Given their ease of use outside the hospital, the lack of monitoring required, and their potential economic savings, LMWHs are emerging as the preferred agents for peri-procedural bridging in many patients [37].

Most published studies employing LMWHs pre-procedure have used enoxaparin dosed at either $1 \mathrm{mg} / \mathrm{kg}$ twice daily or a lower dose of $0.5 \mathrm{mg} / \mathrm{kg}$ twice daily. Enoxaparin $0.5 \mathrm{mg} / \mathrm{kg}$ twice daily is being used with increasing frequency due to the lower rate of femoral vascular complications when this dosing regimen is employed [10,27,30,31]. The last dose of LMWH should be given at least 24 hours prior to the procedure to minimize bleeding events, as recommended in national guidelines [10,24,27,29,37].

\section{How is anticoagulation managed intra-procedurally?}

To prevent thrombus formation, a loading dose of UFH (100 U/kg) followed by a continuous infusion $(10 \mathrm{U} / \mathrm{kg} / \mathrm{hr})$ is often utilized. The ACT is monitored until therapeutic or as needed [22]. In a trial of 3 different ACT targets (250-300s, 300-350s, and 350-400s), there was a lower incidence of TE (stroke and TIA) when the two higher ACT targets were 
maintained [11]. Consistent with these data, current guidelines recommend a target ACT of at least 300-350s throughout the procedure [22,25], although additional TE risk factors (e.g. spontaneous echo contrast and marked atrial enlargement) may warrant higher ACT targets of 350-400s [34,38].

\section{How can anticoagulation post-procedure be managed?}

As with direct current cardioversion, the atria are often "stunned" following radiofrequency ablation [16] and anticoagulation may help prevent thrombus formation and, importantly, stroke and TIA. Many centers restart warfarin and/or heparinoids a few hours after the procedure or the evening of the procedure [10,11,18,22,25,27-29,31,32,39-41]. In patients with higher post-procedure bleeding-risk, the initial use of IV UFH until the morning after the procedure followed by a transition to LMWH [10,18,32,40,41] allows for easier and more rapid reversal of anticoagulation if an early bleeding complication occurs.

When transitioning to LMWH and restarting warfarin, enoxaparin may be initially used therapeutically ( $1 \mathrm{mg} / \mathrm{kg}$ twice daily in the first 24 hours post-procedure) and then decreased to $0.5 \mathrm{mg} / \mathrm{kg}$ twice daily until a therapeutic INR is achieved [31,39]. This approach was associated with a reduction in post-procedure femoral vascular complications compared to 5 days of therapeutic enoxaparin (5.7\% vs $1.6 \%$ ). Importantly, there were no procedurerelated TE events in patients who received this lower-dose enoxaparin regimen and a very low incidence of other bleeding events such as hematomas and pseudoaneurysms [31]. Low rates of post-procedure ischemic stroke were also reported in a study of similar design and were higher in patients with a $\mathrm{CHADS}_{2}$ score $\geq 2$ (odds ratio [OR] 7.1 vs. $\mathrm{CHADS}_{2}=0$ ) or a history of stroke (OR 9.5), although the absolute number of events was small (10/721 patients, $1.4 \%)$ [10].

\section{How do we Estimate Individual Bleeding Risk?}

Although antithrombotic therapy effectively reduces TE risk, this benefit comes at the risk of increasing bleeding, especially in higher-risk patients. Several bleeding risk-assessment scales [42-44] may help clinicians determine their patient's risk of bleeding, although none have been validated well enough to recommend the preferential use of one over another.

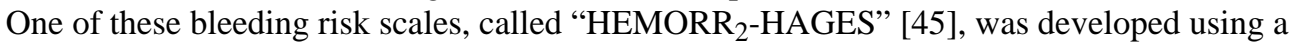
cohort of elderly AF patients prescribed warfarin. HEMORR ${ }_{2} \mathrm{HAGES}$ also identifies subgroups at higher risk of bleeding, including those with liver disease, ethanol abuse, and reduced platelet count or function. Regardless of which risk assessment tool is employed, many of the unique bleeding risks associated with $\mathrm{AF}$ ablation (usually from accessing the vasculature - groin hematomas, retroperitoneal bleeding, hemopericardium, atrioesophageal fistula, etc) are not incorporated into any of these bleeding risk schemes. Rather than relying on just one of these bleeding risk assessment tools, we and others [44] recommend incorporating the presence of these bleeding risk factors (Table 2) into a patient's individual anticoagulation plan.

\section{What is the role of antiplatelet agents pre- and post-ablation?}

Aspirin (ASA) has been used in lower-risk AF patients undergoing ablation, although its precise role as monotherapy and as combination therapy with warfarin remains controversial. In 207 patients with symptomatic AF (85\% had paroxysmal AF and 88\% had a $\mathrm{CHADS}_{2}$ score of $0-1$ ), warfarin was discontinued 3 days prior to ablation and patients received ASA $325 \mathrm{mg}$ daily. Warfarin was resumed on the day of procedure and both medications were continued for 6 weeks. TE events occurred in 2 patients $(0.97 \%)$ within 8 days post-procedure, both with subtherapeutic INRs, and two patients had small groin hematomas post-procedure (0.97\%) [28]. Thus, ASA may be an alternative to LMWH 
bridging to warfarin in AF patients with a low $\mathrm{CHADS}_{2}$ score and paroxysmal AF, although more data is clearly needed. ASA as monotherapy post-procedure may also be safe and effective in carefully selected low-risk AF patients (i.e. $\mathrm{CHADS}_{2}$ score of $0-1$ and paroxysmal AF) [41], although the temporarily higher TE risk post-ablation may warrant warfarin as the preferred agent.

\section{How long should patients remain anticoagulated after ablation?}

The optimal duration of anticoagulation post-ablation ranges from one to six months depending on several variables, including stroke risk factors, $\mathrm{CHADS}_{2}$ score, [22,25,35], type of pre-procedure AF (persistent or paroxysmal) [28], and AF recurrence post-procedure $[11,28,41,46,47]$. Based on the high rate of asymptomatic AF after ablation [48] as well as the increased failure rates in patients with long-standing $\mathrm{AF}$ and/or those who have undergone multiple procedures $[32,35,46]$, the duration of anticoagulation should be based largely on a patient's TE risk factors (i.e. $\mathrm{CHADS}_{2}$ score), as recommended in published guidelines [22,25]. The Venice Chart International Consensus Document on Atrial Fibrillation Ablation recommends that patients complete 3-6 months of warfarin. If the patient's CHADS2 score is $\geq 2$, indefinite warfarin is recommended. For lower risk patients, either ASA or warfarin $\left(\mathrm{CHADS}_{2}=1\right)$ or ASA or no therapy $\left(\mathrm{CHADS}_{2}=0\right)$ are recommended [25]. Similarly, the Heart Rhythm Society/European Heart Rhythm Association/European Cardiac Arrhythmia Society guidelines recommend at least 2 months of warfarin (INR 2-3) post-ablation followed by a duration of anticoagulation based on the patient's stroke risk factors [22]. Both guidelines recommend continuing warfarin indefinitely in any patients with a CHADS 2 score $\geq 2$ [22,25]. Interestingly, a recent study by Dagres and colleagues evaluated real-world adherence to these guidelines. The investigators found that $\mathrm{AF}$ recurrence was a more significant predictor for patients remaining on oral anticoagulation at 12 months post-ablation than the patient's $\mathrm{CHADS}_{2}$ score, possibly leading to overtreatment of low-risk patients, and undertreatment of high-risk patients [49].

\section{Conclusions}

Until large, adequately powered, randomized-controlled trials are available, practitioners must use the best available data and recommendations from consensus guidelines to understand how to safely and effectively reduce TE risk in AF patients undergoing ablation (Tables 3 and 4). Although no conclusive recommendations can be made, published guidelines and data support therapeutic anticoagulation (INR 2-3) for 3 weeks prior to ablation and use of IV UFH during the ablation itself (target ACT of at least 300-350s). Warfarinmay either be continued through the ablation or stopped 2-5 days prior to the ablation, depending on practitioner preference and institutional protocols. If the latter approach is chosen, the authors recommend a pre-ablation bridging strategy of enoxaparin 1 $\mathrm{mg} / \mathrm{kg}$ twice daily unless the patient's bleeding risk dictates using the lower dose regimen (0.5 mg/kg twice daily).

Bridging management strategies post-ablation are even less well standardized or studied, and current practice patterns are based largely on single-center experiences in smaller, nonrandomized studies. No single strategy (either drug or dosing) has been shown to be unequivocally superior and LMWHs, IV UFH, and/or antiplatelet agents may be used until patients are therapeutic on warfarin. For lower risk patients $\left(\mathrm{CHADS}_{2} 0-1\right)$, either warfarin or ASA may be utilized without bridging with a heparinoid. In higher TE risk patients (i.e. $\mathrm{CHADS}_{2}$ score $\geq 2$ ), we recommend the use of therapeutic anticoagulation with either enoxaparin (1 mg/kg twice daily) or IV UFH for the first 12-24 hours. This recommendation places a larger emphasis on preventing TE events post-procedure and should be used thoughtfully, given the potential risk of bleeding. In patients with bleeding risk factors, 
enoxaparin may be subsequently reduced to $0.5 \mathrm{mg} / \mathrm{kg}$ until the INR is therapeutic, although the efficacy and pharmacology of this lower dosing regimen in stroke prevention has not been well studied and should be considered unproven until adequately powered and designed studies have been published. In accordance with national guidelines, warfarin should be continued post-ablation for a minimumof 2 months and then indefinitely in patients with a $\mathrm{CHADS}_{2}$ score $\geq 2$.

\section{Appendix A}

Table 1

Clinical Trials Evaluating Peri-Procedural Anticoagulation for Atrial Fibrillation Ablation.

\begin{tabular}{|c|c|c|c|c|c|c|}
\hline Authors & Patients & Pre-Procedural AC & $\begin{array}{l}\text { Intra- } \\
\text { Procedural } \\
\text { AC }\end{array}$ & Post-Procedural AC & Bleeding & Stroke/TIA \\
\hline $\begin{array}{l}\text { Schmidt, et } \\
\text { al } 2009 \text { [33] }\end{array}$ & $\begin{array}{l}\mathrm{n}=194 \\
\text { Follow-up: }\end{array}$ & $\begin{array}{l}\text { Warfarin or } \\
\text { phenprocoumon } \\
\text { either continued } \\
\text { pre- } \\
\text { procedure or started } \\
3 \text { days } \\
\text { prior to procedure } \\
\text { Controls }(\mathrm{n}=107) \text { : } \\
\text { INR } \\
<2 \text { day of procedure } \\
\text { Cases }(\mathrm{n}=87): \text { INR } \\
\geq 2 \text { day } \\
\text { of procedure } \\
\text { Procedure } \\
\text { postponed if INR } \\
>3.5 \text { day of } \\
\text { procedure }\end{array}$ & ACT $350-450$ & $\begin{array}{l}\text { Controls: enoxaparin } \\
0.5 \\
\mathrm{mg} / \mathrm{kg} \text { BID started } \\
6-8 \\
\text { hours post-procedure } \\
\text { and continued until } \\
\text { therapeutic INR } \\
\text { achieved } \\
\text { Warfarin or } \\
\text { phenprocoumon } \\
\text { restarted in all } \\
\text { patients } \\
\text { PM of procedure }\end{array}$ & $\begin{array}{l}\text { Minor vascular } \\
\text { complications } a \\
\text { Controls: } \mathrm{n}=7 \\
(6.5 \%) \\
\text { Cases: } \mathrm{n}=5 \\
(5.8 \%) \\
\mathrm{p}=\mathrm{NS} \\
\text { Major vascular } \\
\text { complications } b \text { : } \\
\text { Controls: } \mathrm{n}=1 \\
(0.93 \%) \\
\text { Cases: } \mathrm{n}=1 \\
(1.2 \%)\end{array}$ & $\begin{array}{l}\text { Controls: } \mathrm{n}=0 \\
\text { Cases: } \mathrm{n}=1 \\
(1.2 \%)\end{array}$ \\
\hline $\begin{array}{l}\text { Bunch, et al } \\
2009[41]\end{array}$ & $\begin{array}{l}\mathrm{n}=630 \\
\mathrm{CHADS}_{2} \text { score } \\
0-1: 20 \% \\
\mathrm{CHADS}_{2} \text { score } \\
\geq 2: 80 \% \\
\text { Mean follow- } \\
\text { up: } \\
327 \pm 368 \\
\text { days }\end{array}$ & NR & NR & $\begin{array}{l}\text { UFH } 4 \text { hours post- } \\
\text { sheath } \\
\text { removal and } \\
\text { continuing } \\
\text { for } 24 \text { hours } \\
\text { If CHADS } 2 \text { score } \\
0-1=\mathrm{ASA}^{2} 325 \mathrm{mg} \\
\text { daily } \\
\text { (n= 123) } \\
\text { If CHADS } 2 \text { score 2: } \\
\text { LMWH } \\
\text { (dose NR) + warfarin } \\
\text { (n= 507) }\end{array}$ & NR & $\begin{array}{l}\text { ASA group }= \\
0 \% \\
\text { Warfarin group } \\
=0.4 \% \\
\mathrm{p}=\mathrm{NS}\end{array}$ \\
\hline $\begin{array}{l}\text { Prudente, et } \\
\text { al } 2009 \text { [31] }\end{array}$ & $\begin{array}{l}\mathrm{n}=539 \\
(603 \\
\text { procedures }) \\
\text { Mean follow- } \\
\text { up: } \\
1 \text { month }\end{array}$ & $\begin{array}{l}\text { Warfarin D/C } 4 \\
\text { days pre- } \\
\text { procedure to } \\
\text { achieve } \\
\text { INR }<2\end{array}$ & ACT $300-350$ & $\begin{array}{l}\text { Warfarin re-started } \\
4-6 \\
\text { hours post-procedure } \\
\text { enoxaparin } 1 \mathrm{mg} / \mathrm{kg} \\
\text { at } \\
4 \text { hours post- } \\
\text { procedure } \\
\text { and the next } \mathrm{AM} \\
\text { Protocol } A(\mathrm{n}=263) \text { : } \\
\text { enoxaparin } 1 \mathrm{mg} / \mathrm{kg} \\
\text { BID } \\
\times 5 \text { additional days } \\
\text { Protocol } B(\mathrm{n}=85) \text { : } \\
\text { enoxaparin } 1 \mathrm{mg} / \mathrm{kg} \\
\text { BID } \\
\times 3 \text { additional days } \\
\text { Protocol } C(\mathrm{n}= \\
255) \text { : } \\
\text { enoxaparin } 0.5 \mathrm{mg} / \\
\text { kg BID } \\
\times 3 \text { additional days }\end{array}$ & $\begin{array}{l}\text { Femoral vascular } \\
\text { complications: } \\
\text { Protocol A: } 5.7 \% \\
\text { Protocol B: } 2.4 \% \\
\text { Protocol C: } 1.6 \% \\
\text { p }<0.03 \\
\text { (comparing A to } \\
\text { B + C } \\
\text { or A to C) }\end{array}$ & $\begin{array}{l}\text { Protocol A: } \mathrm{n}= \\
1(0.38 \%) \\
\text { Protocol } B: \mathrm{n}= \\
0 \\
\text { Protocol C: } \mathrm{n}= \\
0\end{array}$ \\
\hline $\begin{array}{l}\text { Scherr, et al } \\
2009[10]\end{array}$ & $\begin{array}{l}\mathrm{n}=579 \\
(721 \\
\text { procedures })\end{array}$ & $\begin{array}{l}\text { Warfarin (INR 2-3) } \\
\text { for at }\end{array}$ & ACT $300-400$ & $\begin{array}{l}\text { Warfarin + UFH } \\
\text { initiated } \\
6 \text { hours post-sheath }\end{array}$ & $\begin{array}{l}\text { Pericardial } \\
\text { effusions } \\
n=9 / 721(1.2 \%)\end{array}$ & $\begin{array}{l}\text { Within } \leq 30 \\
\text { days post- }\end{array}$ \\
\hline
\end{tabular}




\begin{tabular}{|c|c|c|c|c|c|c|}
\hline Authors & Patients & Pre-Procedural AC & $\begin{array}{l}\text { Intra- } \\
\text { Procedural } \\
\text { AC }\end{array}$ & Post-Procedural AC & Bleeding & Stroke/TIA \\
\hline & $\begin{array}{l}\text { CHADS }_{2} \text { score } \\
0-1: \\
580 / 721 \text { (79)\% } \\
\text { CHADS } 2 \text { score } \\
\geq 2: \\
\text { 152/721 (21\%) } \\
\text { Follow-up: } 3 \\
\text { months }\end{array}$ & $\begin{array}{l}\text { least } 4 \text { weeks pre- } \\
\text { ablation } \\
\text { Warfarin D/C } 5 \\
\text { days pre- } \\
\text { procedure, and } \\
\text { bridged } \\
\text { with enoxaparin } \\
0.5-1 \mathrm{mg} / \mathrm{kg} \text { BID } g\end{array}$ & & $\begin{array}{l}\text { removal } \\
\text { UFH D/C and } \\
\text { enoxaparin } \\
0.5-1 \mathrm{mg} / \mathrm{kg} \mathrm{BID}{ }^{c} \\
\text { started } \\
\text { the following AM } \\
\text { and } \\
\text { continued until INR } \\
>2 \\
\text { Warfarin continued } \\
\text { for at } \\
\text { least } 3 \text { months }\end{array}$ & $\begin{array}{l}\text { Femoral vascular } \\
\text { complications } \\
\mathrm{n}=11 / 721 \\
(1.5 \%)\end{array}$ & $\begin{array}{l}\text { procedure: } \mathrm{n}= \\
10 / 721 \\
(1.4 \%)(9 \text { were } \\
\text { within } 24 \\
\text { hours of } \\
\text { procedure, } 1 \\
\text { within } 6 \text { days of } \\
\text { procedure) } \\
\text { CHADS }_{2} \text { score } \\
0: 0.3 \% \\
\text { CHADS } \\
1: 1.0 \% \\
\text { CHADS } \\
\geq 2: 4.7 \% \\
\text { score } \\
\text { s. }\end{array}$ \\
\hline $\begin{array}{l}\text { Mortada, et } \\
\text { al } 2008[28]\end{array}$ & $\begin{array}{l}\mathrm{n}=207 \\
\text { CHADS } \\
2 \text { score } \\
0-1: \\
88 \% \\
\text { CHADS }_{2} \text { score } \\
\geq 2: \\
12 \% \\
\text { Mean follow- } \\
\text { up: } \\
24 \pm 6 \text { months }\end{array}$ & $\begin{array}{l}\text { Warfarin D/C } 3 \\
\text { days pre- } \\
\text { procedure, then } \\
\text { ASA } \\
325 \mathrm{mg} \text { daily } \times 3 \\
\text { days } \\
\text { Need } \\
\text { INR } \leq 2\end{array}$ & ACT $300-350$ & $\begin{array}{l}\text { Warfarin (INR 2-3) } \\
\text { and } \\
\text { ASA restarted day of } \\
\text { procedure and } \\
\text { continued } \\
\text { for } 6 \text { weeks } \\
\text { Warfarin continued } \\
\text { beyond } \\
6 \text { weeks only if pt } \\
\text { had AF } \\
\text { recurrence or prior } \\
\text { history } \\
\text { of persistent AF } \\
\text { If no AF recurrence } \\
\text { at } 6 \\
\text { months, warfarin D/ } \\
\text { C } \\
\text { ASA continued for at } \\
\text { least } \\
6 \text { months in all } \\
\text { patients }\end{array}$ & $\begin{array}{l}\text { Groin hematoma } \\
\mathrm{n}=2(0.97 \%) \\
\text { Pericardial } \\
\text { effusion } \\
\mathrm{n}=2(0.97 \%)\end{array}$ & $\begin{array}{l}\mathrm{n}=2(0.97 \%) \\
\text { CHADS } \\
1,2 \\
\text { Both events } \\
\text { occurred within } \\
8 \text { days of } \\
\text { procedure, both } \\
\text { with } \\
\text { subtherapeutic } \\
\text { INR }\end{array}$ \\
\hline $\begin{array}{l}\text { Rossillo, et } \\
\text { al } 2008 \text { [47] }\end{array}$ & $\begin{array}{l}\mathrm{n}=170 \\
\mathrm{n}=85 \\
\text { undergoing } \\
\text { PVAI } \\
(28 \% \text { low- } \\
\text { medium } \\
\text { stroke risk } f ; \\
72 \% \\
\text { high stroke } \\
\text { risk } f) \\
\mathrm{n}=85 \\
\text { matched } \\
\text { controls } \\
\text { undergoing } \\
\text { DCCV } \\
(24 \% \text { low- } \\
\text { medium } \\
\text { stroke risk } f ; \\
76 \% \\
\text { high stroke } \\
\text { risk } f) \\
\text { Mean follow- } \\
\text { up: } \\
15 \pm 7 \text { months }\end{array}$ & $\begin{array}{l}\text { AC started } 1 \text { month } \\
\text { pre- procedure }\end{array}$ & ACT $350-400$ & $\begin{array}{l}\text { ASA } 325 \mathrm{mg} \times 1 \\
\text { dose given } \\
\text { at the end of } \\
\text { procedure } \\
\text { All patients } \\
\text { discharged } \\
\text { with warfarin } \times 3 \\
\text { months } \\
\text { Warfarin was D/C } \\
\text { after } \\
3 \text { months unless } \\
\text { patient } \\
\text { had one of the } \\
\text { following: } \\
\text { recurrence of AF } \\
>60 \% \text { PV } \\
\text { narrowing Poor atrial } \\
\text { contractility Presence } \\
\text { of } \\
\text { other indications for } \\
\text { AC }\end{array}$ & NR & $\begin{array}{l}\text { PVAI group } \mathrm{n}= \\
1(1.2 \%) \\
(<30 \text { days after } \\
\text { procedure }) \\
\text { Control group } \\
\mathrm{n}=5(5.9 \%) \\
(1<30 \text { days } \\
\text { after procedure, } \\
4>30 \text { days after } \\
\text { procedure })\end{array}$ \\
\hline $\begin{array}{l}\text { Nademanee, } \\
\text { et al } 2008 \\
{[29]}\end{array}$ & $\begin{array}{l}\mathrm{n}=635(1,065 \\
\text { procedures }) \\
\text { Age }>65 \text { or had } \\
\text { at least } 1 \\
\text { stroke } \\
\text { risk factor } \\
\text { Mean follow- } \\
\text { up: } \\
836 \pm 605 \\
\text { days }\end{array}$ & $\begin{array}{l}\text { Warfarin (INR 2-3) } \\
\text { for } \\
\text { at least } 3 \text { weeks pre- } \\
\text { procedure } \\
\text { Warfarin D/C } 4 \\
\text { days } \\
\text { pre-procedure } \\
\text { If persistent or } \\
\text { permanent } \\
\text { AF, bridged with }\end{array}$ & NR & $\begin{array}{l}\text { Warfarin + } \\
\text { enoxaparin } 1 \\
\text { mg/kg BID restarted } \\
\text { immediately } \\
\text { post-procedure } \\
\text { Enoxaparin D/C } 3 \\
\text { days } \\
\text { later } \\
\text { Warfarin D/C after } 3 \\
\text { months if NSR } \\
\text { maintained, }\end{array}$ & $\begin{array}{l}\text { Hemopericardium } \\
\mathrm{n}=9 / 635(1.4 \%) \\
\text { Femoral vascular } \\
\text { complications } \\
\mathrm{n}=13 / 635(2 \%)\end{array}$ & $\begin{array}{l}\mathrm{n}=11 / 517 \\
(2.1 \%) \\
\text { Patients who D/ } \\
\mathrm{C} \text { warfarin } \\
\mathrm{n}=5 / 434 \\
(1.2 \%) \\
\text { Patients who } \\
\text { continued } \\
\text { warfarin } \\
\mathrm{n}=6 / 83(7.2 \%)\end{array}$ \\
\hline
\end{tabular}




\begin{tabular}{|c|c|c|c|c|c|c|}
\hline Authors & Patients & Pre-Procedural AC & $\begin{array}{l}\text { Intra- } \\
\text { Procedural } \\
\text { AC }\end{array}$ & Post-Procedural AC & Bleeding & Stroke/TIA \\
\hline & & $\begin{array}{l}\text { enoxaparin } 1 \mathrm{mg} / \mathrm{kg} \\
\text { BID }\end{array}$ & & $\begin{array}{l}\text { then ASA or } \\
\text { clopidogrel } \\
\text { prescribed }\end{array}$ & & $\begin{array}{l}4 \text { with adequate } \\
\text { INRs } 3 \\
\text { months prior, } 2 \\
\text { had no } \\
\text { documented } \\
\text { INR within } \\
3 \text { months of } \\
\text { event }\end{array}$ \\
\hline $\begin{array}{l}\text { Seow, et al } \\
2007[32]\end{array}$ & $\begin{array}{l}\mathrm{n}=56(86 \\
\text { procedures }) \\
\text { Mean follow- } \\
\text { up: } \\
21.6 \pm 8.8 \\
\text { months }\end{array}$ & $\begin{array}{l}\text { AC D/C 3-5 days } \\
\text { pre- } \\
\text { procedure }\end{array}$ & ACT $300-350$ & $\begin{array}{l}\text { UFH } 4 \text { hours post- } \\
\text { procedure and } \\
\text { continued } \\
\text { until the following } \\
\text { AM, } \\
\text { then changed to } \\
\text { LMWH } \\
\text { until INR >2 } \\
\text { Warfarin re-started } \\
\text { the } \\
\text { following AM }\end{array}$ & $\begin{array}{l}\text { Cardiac } \\
\text { tamponade } \\
\mathrm{n}=1(1.1 \%)\end{array}$ & $\mathrm{n}=2(2.3 \%)$ \\
\hline $\begin{array}{l}\text { Wazni, et al } \\
2007[27]\end{array}$ & $\begin{array}{l}\mathrm{n}=355 \\
\text { Mean follow- } \\
\text { up: } \\
\text { 3-4 months }\end{array}$ & $\begin{array}{l}\text { Groups } 1 \text { and 2: } \\
\text { warfarin D/C } \\
2-3 \text { days prior to } \\
\text { procedure } \\
\text { Group 3: warfarin } \\
\text { continued } \\
\text { without interruption } \\
\text { (INR 2-3.5) }\end{array}$ & ACT $350-450$ & $\begin{array}{l}\text { ASA } 325 \mathrm{mg} \times 1 \\
\text { dose at } \\
\text { the end of procedure } \\
\text { Warfarin restarted } \\
\text { PM of } \\
\text { procedure } \\
\text { Group } 1(\mathrm{n}=105) \text { : } \\
\text { enoxaparin } 1 \mathrm{mg} / \mathrm{kg} \\
\text { BID } \\
\text { until INR >2 } \\
\text { Group } 2(\mathrm{n}=100) \text { : } \\
\text { enoxaparin } 0.5 \mathrm{mg} / \\
\text { kg BID } \\
\text { until INR }>2 \\
\text { Group } 3(\mathrm{n}=150) \text { : } \\
\text { continued warfarin } \\
\text { (INR 2-3.5) }\end{array}$ & $\begin{array}{l}\text { Minor bleeding } d: \\
\text { Group } 1: \mathrm{n}=23 \\
(22 \%) \\
\text { Group 2: } \mathrm{n}=19 \\
(19 \%) \\
\text { Group 3: } \mathrm{n}=8 \\
(5.3 \%) \\
\mathrm{p}<0.001 \\
\text { Major bleeding } e: \\
\text { Group } 1: \mathrm{n}=9 \\
((8.6 \%) \\
\text { Group } 2: \mathrm{n}=0 \\
(0 \%) \\
\text { Group 3: } \mathrm{n}=0 \\
(0 \%) \\
\mathrm{p}<0.001\end{array}$ & $\begin{array}{l}\text { Group } 1: \mathrm{n}=1 \\
(0.95 \%) \\
\text { Group } 2: \mathrm{n}=2 \\
(2 \%) \\
\text { Group } 3: \mathrm{n}=0 \\
\mathrm{p}=\mathrm{NS}\end{array}$ \\
\hline $\begin{array}{l}\text { Oral, et al } \\
2006[30]\end{array}$ & $\begin{array}{l}\mathrm{n}=755 \\
\text { No stroke risk } \\
\text { factors } c: 44 \% \\
\geq 1 \text { stroke risk } \\
\text { factors } c: 56 \% \\
\text { Mean follow- } \\
\text { up: } \\
25 \pm 8 \text { months }\end{array}$ & $\begin{array}{l}\text { Warfarin D/C } 5 \\
\text { days pre- } \\
\text { procedure }\end{array}$ & ACT $300-350$ & $\begin{array}{l}\text { UFH } 1000 \text { units/hour } \\
\text { within } 3 \text { hours of } \\
\text { sheath } \\
\text { removal, continued } \\
\text { until } \\
\text { the following AM, } \\
\text { then } \\
\text { changed to } \\
\text { enoxaparin } \\
0.5 \mathrm{mg} / \mathrm{kg} \text { BID until } \\
\text { INR } \geq 2 \\
\text { Warfarin restarted } \\
\text { PM of } \\
\text { procedure and } \\
\text { continued } \\
\text { for at least } 3 \text { months } \\
\text { ASA } 81-325 \text { mg } \\
\text { daily } \\
\text { indefinitely after } \\
\text { warfarin } \\
\text { D/C }\end{array}$ & $\begin{array}{l}\text { Cerebral } \\
\text { hemorrhage } \\
n=2(0.3 \%)\end{array}$ & $\begin{array}{l}\leq 30 \text { days of } \\
\text { ablation: } \\
\mathrm{n}=7(0.9 \%) \\
(1 \\
\text { intraprocedural, } \\
3 \text { on } \\
\text { enoxaparin with } \\
\text { INR }<2 \text {, } \\
3 \text { off } \\
\text { enoxaparin with } \\
\text { INR } \leq 2) \\
>30 \text { days post- } \\
\text { ablation: } \\
\mathrm{n}=2 \text { (0.3\%) } \\
\text { (both on } \\
\text { warfarin with } \\
\text { INR 2.6-3.2) }\end{array}$ \\
\hline $\begin{array}{l}\text { Wazni, et al } \\
2005[11]\end{array}$ & $\begin{array}{l}\mathrm{n}=785 \\
\text { Follow-up: } \\
12 \text { months }\end{array}$ & $\begin{array}{l}\text { Warfarin (INR 2-3) } \\
\text { D/C } 48 \text { hours pre- } \\
\text { procedure }\end{array}$ & $\begin{array}{l}\text { Group 1: } \\
(\mathrm{n}=194) \\
\text { ACT 250-300 }\end{array}$ & $\begin{array}{l}\text { ASA } \times 1 \text { dose at end } \\
\text { of } \\
\text { procedure }(\text { dose } \mathrm{NR}) \\
\text { Warfarin restarted } \\
\text { day } \\
\text { of procedure }\end{array}$ & $\begin{array}{l}\text { Groin hematoma: } \\
\text { Group } 1: \mathrm{n}=1 \\
\text { Group } 2: \mathrm{n}=2 \\
\text { Group } 3: \mathrm{n}=2\end{array}$ & $\begin{array}{l}\text { Group } 1: \mathrm{n}=7 \\
(3.6 \%) \\
\text { Group } 2: \mathrm{n}=3 \\
(1.7 \%)\end{array}$ \\
\hline $\begin{array}{l}\text { Wazni, et al } \\
2005[11]\end{array}$ & & & $\begin{array}{l}\text { Group 2: } \\
(\mathrm{n}=180) \\
\text { ACT 300-350 } \\
+ \\
\text { Eptifibatide }\end{array}$ & $\begin{array}{l}\text { If history of } \\
\text { persistent } \\
\text { AF: enoxaparin } 0.5 \\
\mathrm{mg} / \mathrm{kg}\end{array}$ & $\begin{array}{l}\text { Pericardial } \\
\text { effusion: } \\
\text { Group 1: } \mathrm{n}=1 \\
\text { Group 2: } \mathrm{n}=1 \\
\text { Group 3: } \mathrm{n}=0\end{array}$ & $\begin{array}{l}\text { Group 3: } \mathrm{n}=2 \\
(0.49 \%) \\
\text { All events were } \\
\text { intra- } \\
\text { procedural }\end{array}$ \\
\hline
\end{tabular}




\begin{tabular}{|c|c|c|c|c|c|c|}
\hline Authors & Patients & Pre-Procedural AC & $\begin{array}{l}\text { Intra- } \\
\text { Procedural } \\
\text { AC }\end{array}$ & Post-Procedural AC & Bleeding & Stroke/TIA \\
\hline & & & $\begin{array}{l}\text { Group 3: } \\
(\mathrm{n}=411) \\
\text { ACT 350-400 }\end{array}$ & $\begin{array}{l}\text { BID until therapeutic } \\
\text { INR } \\
\text { achieved } \\
\text { Warfarin D/C after } \\
4-6 \\
\text { months unless } \\
\text { AF recurrence or } \\
>70 \% \text { PV } \\
\text { narrowing }\end{array}$ & $\begin{array}{l}\text { Cardiac } \\
\text { tamponade: } \\
\text { Group 1: } \mathrm{n}=1 \\
\text { Group 2: } \mathrm{n}=1 \\
\text { Group 3: } \mathrm{n}=0\end{array}$ & \\
\hline
\end{tabular}

$\mathrm{AC}=$ anticoagulation; $\mathrm{ACT}=$ activated clotting time $; \mathrm{AF}=$ atrial fibrillation; $\mathrm{AM}=$ morning; $\mathrm{ASA}=$ aspirin; $\mathrm{BID}=\mathrm{twice}$ daily; $\mathrm{CHADS}_{2}=$ stroke risk stratification system; $\mathrm{D} / \mathrm{C}=$ discontinued; $\mathrm{DCCV}=$ direct current cardioversion; INR = international normalized ratio; LMWH = low-molecular weight heparin; NR = not reported; NSR = normal sinus rhythm; $\mathrm{PM}=$ evening; $\mathrm{PV}=$ pulmonary vein; $\mathrm{PVAI}=$ pulmonary vein antrum isolation; $\mathrm{TIA}=$ transient ischemic attack; UFH = unfractionated heparin. $a$

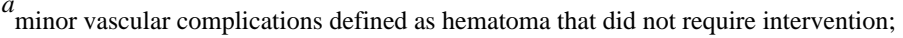

$b$ major vascular complications defined as hematoma that required intervention or bleeding that required blood product transfusions;

${ }^{c}$ congestive heart failure, hypertension, age $>65$, diabetes mellitus, prior stroke or transient ischemic attack;

$d_{\text {hematoma not requiring intervention; }}$

$e$ cardiac tamponade, hematoma requiring intervention, bleeding requiring transfusion;

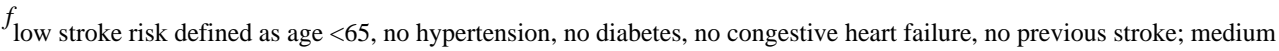
stroke risk defined as age $>65$, no hypertension, no diabetes, no congestive heart failure, no previous stroke; high stroke risk defined as age $>65$ plus hypertension or diabetes or congestive heart failure or previous stroke OR age $>75$ without other risk factors;

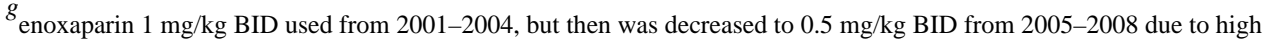
incidence of vascular complications.

\section{References}

1. Fuster V, Rydén LE, Cannom DS, Crijns HJ, et al. European Heart Rhythm Association, Heart Rhythm Society. ACC/AHA/ESC 2006 guidelines for the management of patients with atrial fibrillation-executive summary: a report of the American College of Cardiology/American Heart Association Task Force on Practice Guidelines and the European Society of Cardiology Committee for Practice Guidelines (Writing Committee to Revise the 2001 Guidelines for the Management of Patients With Atrial Fibrillation). J Am Coll Cardiol. 2006; 48:854-906. [PubMed: 16904574]

2. Risk factors for stroke and efficacy of antithrombotic therapy in atrial fibrillation. Analysis of pooled data from five randomized controlled trials. Arch Intern Med. 1994; 154:1449-1457. [PubMed: 8018000]

3. Bornstein N, Corea F, Galllai V, Parnetti L. Heart-brain relationship: atrial fibrillation and stroke. Clin Exp Hypertens. 2002; 24:493-499. [PubMed: 12450224]

4. Stroke Risk in Atrial Fibrillation Working Group. Comparison of 12 risk stratification schemes to predict stroke in patients with nonvalvular atrial fibrillation. Stroke. 2008; 39:1901-1910. [PubMed: 18420954]

5. Gage BF, Waterman AD, Shannon W, Boechler M, Rich MW, Radford MJ. Validation of clinical classification schemes for predicting stroke: results from the National Registry of Atrial Fibrillation. JAMA. 2001; 285:2864-2870. [PubMed: 11401607]

6. Hart RG, Benavente O, McBride R, Pearce LA. Antithrombotic therapy to prevent stroke in patients with atrialfibrillation: a meta-analysis. Ann Intern Med. 1999; 131:492-501. [PubMed: 10507957]

7. Gage BF, van Walraven C, Pearce L, Hart RG, Koudstaal PJ, Boode BS, et al. Selecting patients with atrial fibrillation for anticoagulation: stroke risk stratification in patients taking aspirin. Circulation. 2004; 110:2287-2292. [PubMed: 15477396] 
8. Lip G, Nieuwlaat R, Pisters R, Lane D, Crijns H. Refining clinical risk stratification for predicting stroke and thromboembolism in atrial fibrillation using a novel risk factor based approach: The Euro Heart Survey on Atrial Fibrillation. Chest Sep. 2009; 17 [Electronic publication ahead of print].

9. Rietbrock S, Heeley E, Plumb J, van Staa T. Chronic atrial fibrillation: Incidence, prevalence, and prediction of stroke using the Congestive heart failure, Hypertension, Age > 75, Diabetes mellitus, and prior Stroke or transient ischemic attack (CHADS2) risk stratification scheme. Am Heart J. 2008; 156:57-64. [PubMed: 18585497]

10. Scherr D, Sharma K, Dalal D, Spragg D, Chilukuri K, Cheng A, et al. Incidence and Predictors of Periprocedural Cerebrovascular Accident in Patients Undergoing Catheter Ablation of Atrial Fibrillation. 2009; 20(12):1357-1363.

11. Wazni OM, Rossillo A, Marrouche NF, Saad EB, Martin DO, Bhargava M, et al. Embolic events and char formation during pulmonary vein isolation in patients with atrial fibrillation: impact of different anticoagulation regimens and importance of intracardiac echo imaging. J Cardiovasc Electrophysiol. 2005; 16:576-581. [PubMed: 15946352]

12. Dorwarth U, Fiek M, Remp T, Reithmann C, Dugas M, Steinbeck G, et al. Radiofrequency catheter ablation: different cooled and noncooled electrode systems induce specific lesion geometries and adverse effects profiles. Pacing Clin Electrophysiol. 2003; 26:1438-1445. [PubMed: 12914619]

13. Ren JF, Marchlinski FE, Callans DJ. Left atrial thrombus associated with ablation for atrial fibrillation: identification with intracardiac echocardiography. J Am Coll Cardiol. 2004; 43:18611867. [PubMed: 15145112]

14. Luther T, Mackman N. Tissue factor in the heart. Multiple roles in hemostasis, thrombosis, and inflammation. Trends Cardiovasc Med. 2001; 11:307-312. [PubMed: 11728878]

15. Demolin JM, Eick OJ, Münch K, Koullick E, Nakagawa H, Wittkampf FH. Soft thrombus formation in radiofrequency catheter ablation. Pacing Clin Electrophysiol. 2002; 25:1219-1222. [PubMed: 12358173]

16. Sparks PB, Jayaprakash S, Vohra JK, Mond HG, Yapanis AG, Grigg LE, et al. Left atrial "stunning" following radiofrequency catheter ablation of chronic atrial flutter. J Am Coll Cardiol. 1998; 32:468-475. [PubMed: 9708477]

17. Cappato R, Calkins H, Chen SA, Davies W, Iesaka Y, Kalman J, et al. Worldwide survey on the methods, efficacy, and safety of catheter ablation for human atrial fibrillation. Circulation. 2005; 111:1100-1105. [PubMed: 15723973]

18. Oral H, Chugh A, Ozaydin M, Good E, Fortino J, Sankaran S, et al. Risk of thromboembolic events after percutaneous left atrial radiofrequency ablation of atrial fibrillation. Circulation. 2006; 114:759-765. [PubMed: 16908760]

19. Bertaglia E, Zoppo F, Tondo C, Colella A, Mantovan R, Senatore G, et al. Early complications of pulmonary vein catheter ablation for atrialfibrillation: amulticenter prospective registry on procedural safety. Heart Rhythm. 2007:1265-1271. [PubMed: 17905330]

20. Sakurai K, Hirai T, Nakagawa K, Kameyama T, Nozawa T, Asanoi H, et al. Left atrial appendage function and abnormal hypercoagulability in patients with atrial flutter. Chest. 2003; 124:16701674. [PubMed: 14605033]

21. Singer DE, Albers GW, Dalen JE, Fang MC, Go AS, Halperin JL, et al. Antithrombotic therapy in atrial fibrillation: American College of Chest Physicians Evidence-Based Clinical Practice Guidelines (8th Edition). Chest. 2008; 133:546S-592S. [PubMed: 18574273]

22. Calkins H, Brugada J, Packer DL, Cappato R, Chen SA, Crijns HJ, et al. HRS/EHRA/ECAS expert consensus statement on catheter and surgical ablation of atrial fibrillation: recommendations for personnel, policy, procedures and follow-up. A report of the Heart Rhythm Society (HRS) Task Force on Catheter and Surgical Ablation of Atrial Fibrillation developed in partnership with the European Heart Rhythm Association (EHRA) and the European Cardiac Arrhythmia Society (ECAS); in collaboration with the American College of Cardiology (ACC), American Heart Association (AHA), and the Society of Thoracic Surgeons (STS). Endorsed and approved by the governing bodies of the American College of Cardiology, the American Heart Association, the European Cardiac Arrhythmia Society, the European Heart Rhythm Association, the Society of Thoracic Surgeons, and the Heart Rhythm Society. Europace. 2007; 9:335-379. [PubMed: 17599941] 
23. Stabile G, Bertaglia E, Senatore G, De Simone A, Zoppo F, Donnici G, et al. Catheter ablation treatment in patients with drug-refractory atrial fibrillation: a prospective, multi-centre, randomized, controlled study (Catheter Ablation For The Cure Of Atrial Fibrillation Study). Eur Heart J. 2006; 27:216-221. [PubMed: 16214831]

24. Scherr D, Dalal D, Chilukuri K, Dong J, Spragg D, Henrikson CA, et al. Incidence and predictors of left atrial thrombus prior to catheter ablation of atrial fibrillation. J Cardiovasc Electrophysiol. 2009; 20:379-384. [PubMed: 19017348]

25. Natale A, Raviele A, Arentz T, Calkins H, Chen SA, Haïssaguerre M, et al. Venice Chart international consensus document on atrial fibrillation ablation. J Cardiovasc Electrophysiol. 2007; 18:560-580. [PubMed: 17456138]

26. Scheinman M, Calkins H, Gillette P, Klein R, Lerman BB, Morady F, et al. NASPE policy statement on catheter ablation: personnel, policy, procedures, and therapeutic recommendations. Pacing Clin Electrophysiol. 2003; 26:789-799. [PubMed: 12698688]

27. Wazni OM, Beheiry S, Fahmy T, Barrett C, Hao S, Patel D, et al. Atrial fibrillation ablation in patients with therapeutic international normalized ratio: comparison of strategies of anticoagulation management in the periprocedural period. Circulation. 2007; 116:2531-2534. [PubMed: 17998456]

28. Mortada ME, Chandrasekaran K, Nangia V, Dhala A, Blanck Z, Cooley R, et al. Periprocedural anticoagulation for atrial fibrillation ablation. J Cardiovasc Electrophysiol. 2008; 19:362-366. [PubMed: 18284509]

29. Nademanee K, Schwab MC, Kosar EM, Karwecki M, Moran MD, Visessook N, et al. Clinical outcomes of catheter substrate ablation for high-risk patients with atrial fibrillation. J Am Coll Cardiol. 2008; 51:843-849. [PubMed: 18294570]

30. Oral H, Chugh A, Ozaydin M, Good E, Fortino J, Sankaran S, et al. Risk of thromboembolic events after percutaneous left atrial radiofrequency ablation of atrial fibrillation. Circulation. 2006; 114:759-765. [PubMed: 16908760]

31. Prudente LA, Moorman JR, Lake D, Xiao Y, Greebaum H, Mangrum JM, et al. Femoral vascular complications following catheter ablation of atrial fibrillation. J Interv Card Electrophysiol. 2009; 26:59-64. [PubMed: 19387807]

32. Seow SC, Lim TW, Koay CH, Ross DL, Thomas SP. Efficacy and late recurrences with wide electrical pulmonary vein isolation for persistent and permanent atrial fibrillation. Europace. 2007; 9:1129-1133. [PubMed: 17923474]

33. Schmidt M, Segerson NM, Marschang H, Akoum N, Rittger H, Clifford SM, et al. Atrial fibrillation ablation in patients with therapeutic international normalized ratios. Pacing Clin Electrophysiol. 2009; 32:995-999. [PubMed: 19659617]

34. Ren JF, Marchlinski FE, Callans DJ, Gerstenfeld EP, Dixit S, Lin D, et al. Increased intensity of anticoagulation may reduce risk of thrombusduring atrial fibrillation ablation procedures in patients with spontaneous echo contrast. J Cardiovasc Electrophysiol. 2005; 16:474-477. [PubMed: 15877616]

35. Shah AN, Mittal S, Sichrovsky TC, Cotiga D, Arshad A, Maleki K, et al. Long-term outcome following successful pulmonary vein isolation: pattern and prediction of very late recurrence. $\mathrm{J}$ Cardiovasc Electrophysiol. 2008; 19:661-667. [PubMed: 18284502]

36. He H, Kang J, Tao H, Zhen B, Zhang M, Long D, et al. Conventional oral anticoagulation may not replace prior transesophageal echocardiography for the patients with planned catheter ablation for atrial fibrillation. J Interv Card Electrophysiol. 2009; 24:19-26. [PubMed: 18982437]

37. Douketis JD, Berger PB, Dunn AS, Jaffer AK, Spyropoulos AC, Becker RC, et al. The perioperative management of antithrombotic therapy: American College of Chest Physicians Evidence-Based Clinical Practice Guidelines (8th Edition). Chest. 2008; 133:299S-339S. [PubMed: 18574269]

38. Pappone C, Santinelli V. The who, what, why, and how-to guide for circumferential pulmonary vein ablation. J Cardiovasc Electrophysiol. 2004; 15:1226-1230. [PubMed: 15485455]

39. Shea JB. Anticoagulation practice in cardiac electrophysiology. Heart Rhythm. 2006; 3:372-374. [PubMed: 16500316] 
40. Oral H, Pappone C, Chugh A, Good E, Bogun F, Pelosi F Jr, et al. Circumferential pulmonary-vein ablation for chronic atrial fibrillation. N Engl J Med. 2006; 354:934-941. [PubMed: 16510747]

41. Bunch TJ, Crandall BG, Weiss JP, May HT, Bair TL, Osborn JS, et al. Warfarin Is Not Needed in Low-Risk Patients Following Atrial Fibrillation Ablation Procedures. J Cardiovasc Electrophysiol. 2009; 20:988-993. [PubMed: 19473299]

42. Wells PS, Forgie MA, Simms M, Greene A, Touchie D, Lewis G, et al. The outpatient bleeding risk index: validation of a tool for predicting bleeding rates in patients treated for deep venous thrombosis and pulmonary embolism. Arch Intern Med. 2003; 163:917-920. [PubMed: 12719200]

43. Kuijer PM, Hutten BA, Prins MH, Büller HR. Prediction of the risk of bleeding during anticoagulant treatment for venous thromboembolism. Arch Intern Med. 1999; 159:457-460. [PubMed: 10074953]

44. Kearon C, Ginsberg JS, Kovacs MJ, Anderson DR, Wells P, Julian JA, et al. Comparison of lowintensity warfarin therapy with conventional-intensity warfarin therapy for long-term prevention of recurrent venous thromboembolism. N Engl JMed. 2003; 349:631-639. [PubMed: 12917299]

45. Gage BF, Yan Y, Milligan PE, Waterman AD, Culverhouse R, Rich MW, et al. Clinical classification schemes for predicting hemorrhage: results from the National Registry of Atrial Fibrillation (NRAF). Am Heart J. 2006; 151:713-719. [PubMed: 16504638]

46. Katritsis D, Wood MA, Giazitzoglou E, Shepard RK, Kourlaba G, Ellenbogen KA. Long-term follow-up after radiofrequency catheter ablation for atrial fibrillation. Europace. 2008; 10:419424. [PubMed: 18287087]

47. Rossillo A, Bonso A, Themistoclakis S, Riccio G, Madalosso M, Corrado A, et al. Role of anticoagulation therapy after pulmonary vein antrum isolation for atrial fibrillation treatment. $\mathrm{J}$ Cardiovasc Med (Hagerstown). 2008; 9:51-55. [PubMed: 18268419]

48. Hindricks G, Piorkowski C, Tanner H, Kobza R, Gerds-Li JH, Carbucicchio C, et al. Perception of atrial fibrillation before and after radiofrequency catheter ablation: relevance of asymptomatic arrhythmia recurrence. Circulation. 2005; 112:307-313. [PubMed: 16009793]

49. Dagres N, Hindricks G, Kottkamp H, Sommer P, Gaspar T, Bode K, et al. Real-life anticoagulation treatment of atrial fibrillation after catheter ablation: Possible overtreatment of low-risk patients. Thromb Haemost. 2009; 102:754-758. [PubMed: 19806262] 
Table 1

The $\mathrm{CHADS}_{2}$ Scoring System.

\begin{tabular}{|c|c|c|}
\hline \multicolumn{3}{|c|}{ A. Independent stroke risk factors comprising the $\mathrm{CHADS}_{2}$ score. [5] } \\
\hline & Independent Stroke Risk Factors & Points \\
\hline $\mathrm{C}$ & Congestive Heart Failure & 1 \\
\hline $\mathrm{H}$ & Hypertension & 1 \\
\hline A & $\underline{\text { Age }}>75$ years & 1 \\
\hline $\mathrm{D}$ & Diabetes Mellitus & 1 \\
\hline $\mathrm{S}_{2}$ & 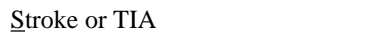 & 2 \\
\hline \multicolumn{3}{|c|}{ B. $\mathrm{CHADS}_{2}$ score with corresponding risk and prophylactic anticoagulation recommendations. [8] } \\
\hline $\mathrm{CHADS}_{2}$ Score & Stroke Risk & Recommended Anticoagulation \\
\hline 0 & Low & ASA $81-325 \mathrm{mg}$ daily \\
\hline 1 & Intermediate & $\begin{array}{l}\text { ASA } 81-325 \mathrm{mg} \text { daily } \text { or warfarin INR 2-3 (dependent upon patient preference and } \\
\text { risk/benefit ratio) }\end{array}$ \\
\hline$\geq 2$ & High & Warfarin INR 2-3 (unless contraindicated) \\
\hline
\end{tabular}

$\mathrm{ASA}=$ aspirin; $\mathrm{INR}=$ international normalized ratio; $\mathrm{TIA}=$ transient ischemic attack. 


\section{Table 2}

Major risk factors associated with hemorrhage on anticoagulant therapy [42-44].

\begin{tabular}{ll}
\hline Increased age ( $>65$ years) & Medication non-adherence, poor clinical follow-up \\
Female gender & Prior stroke or intracerebral hemorrhage \\
Diabetes mellitus & Gastrointestinal bleeding or peptic ulcer disease \\
Active malignancy & Bleeding diathesis (thrombocytopenia, coagulation defects) \\
Hypertension, uncontrolled & Concurrent aspirin or NSAID administration \\
Liver disease & INR $>3.0$ and INR instability \\
Chronic kidney disease & Pretreatment INR $>1.2$ \\
Anemia & Prior major hemorrhage on therapeutic warfarin (INR 2-3) \\
\hline
\end{tabular}

INR=international normalized ratio; NSAID=non-steroidal anti-inflammatory drugs. 
Table 3

Summary of Peri-Procedural Anticoagulation Recommendations for Atrial Fibrillation Ablation.

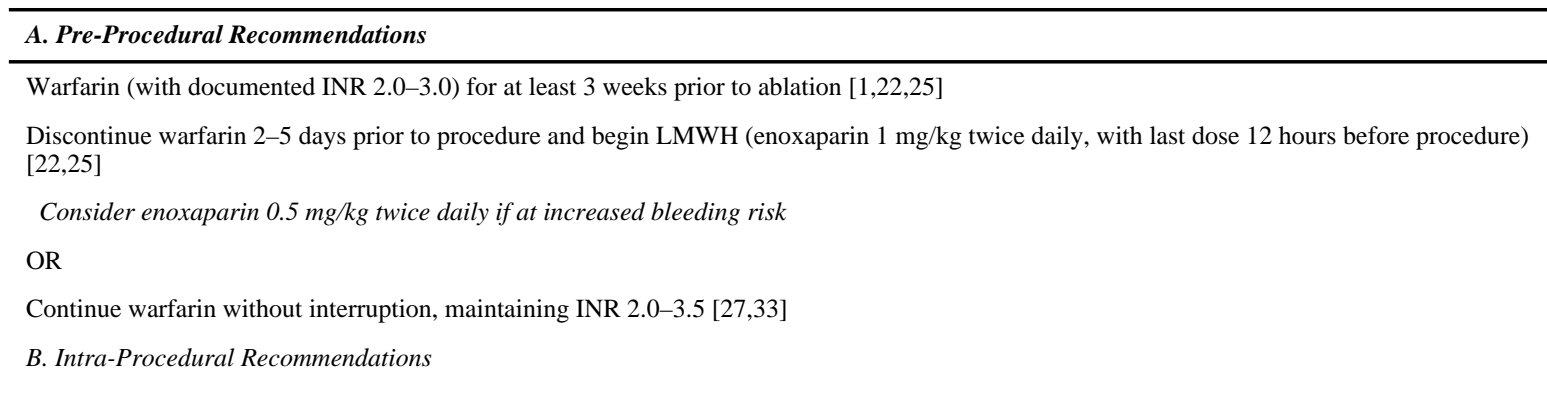

UFH loading dose (100-140 U/kg) prior to, or immediately upon, transeptal puncture [22,25]

UFH continuous infusion (10-18 U/kg/hr) titrated to target ACT [22,25]

- Maintain ACT target of at least 300-350 seconds

- Consider higher ACT target of 350-400 seconds if spontaneous echo contrast or significant atrial enlargement [22,33,34]

Monitor ACT at 10-15 minute intervals until therapeutic, then at 30 minute intervals

Discontinue UFH infusion once all catheters are removed from left atrium [25]

C. Post-Procedural Recommendations

Re-start warfarin evening of procedure or the following morning [22,25]

Re-start LMWH (enoxaparin 0.5-1 mg/kg twice daily) beginning the evening of procedure, and continuing until INR $\geq 2.0[22,25,33]$

Consider using enoxaparin $1 \mathrm{mg} / \mathrm{kg}$ for the first 1 or 2 doses and then decreasing to $0.5 \mathrm{mg} / \mathrm{kg}$ twice daily until therapeutic INR achieved $[22,31,39]$

OR

If warfarin was not interrupted, continue warfarin maintaining INR 2.0-3.5 [27,33]

Continue warfarin for at least 2-3 months post-ablation. Beyond that, continuation of warfarin depends on the patient's stroke risk factors (CHADS score $\geq 2$ should continue warfarin indefinitely) $[22,25]$

INR=international normalized ratio; LMWH=low-molecular weight heparin; UFH=Unfractionated Heparin; ACT=Activated Clotting Time.

Note: These recommendations are not a consensus statement and are limited by the lack of data from randomized, controlled, adequatelypowered studies. 
Table 4

Summary of the Consensus Guidelines Statements on Peri-Procedural Anticoagulation in Atrial Fibrillation Ablation.

\begin{tabular}{|c|c|c|c|}
\hline & $\begin{array}{l}\text { ACC/AHA/ESC } \\
\text { Guidelines for the } \\
\text { Management of Patients } \\
\text { with Atrial } \\
\text { Fibrillation (2006) [1] }\end{array}$ & $\begin{array}{l}\text { Venice Chart International Consensus } \\
\text { Document on Atrial Fibrillation Ablation } \\
\text { (2007) [25] }\end{array}$ & $\begin{array}{l}\text { HRS/EHRA/ECAS Expert Consensus } \\
\text { Statement } \\
\text { on Catheter and Surgical Ablation of } \\
\text { Atrial } \\
\text { Fibrillation: Recommendations for } \\
\text { Personnel, } \\
\text { Policy, Procedures and Follow-Up } \\
(2007)[22]\end{array}$ \\
\hline \multirow[t]{2}{*}{$\begin{array}{l}\text { Atrial Fibrillation } \\
\text { Stroke Risk } \\
\text { Stratification } \\
\text { Scheme Used }\end{array}$} & $\begin{array}{l}\text { No risk factors }{ }^{a}=\text { ASA } 81- \\
325 \text { mg daily } 1 \text { moderate } \\
\text { risk factor=ASA } 81-325 \\
\text { mg daily or warfarin (INR } \\
2-3 \text { ) }\end{array}$ & $\mathrm{CHADS}_{2}$ scoring system & $\mathrm{CHADS}_{2}$ scoring system \\
\hline & $\begin{array}{l}\text { Any high risk factor or }>1 \\
\text { moderate risk factor }= \\
\text { warfarin }(\text { INR } 2-3)\end{array}$ & & \\
\hline \multirow[t]{2}{*}{$\begin{array}{l}\text { Pre-ablation } \\
\text { Recommendations }\end{array}$} & $\begin{array}{l}\text { No specific } \\
\text { recommendations regarding } \\
\text { AF ablation, but } \\
\text { recommend anticoagulation } \\
\text { for } 3 \text { weeks prior to } \\
\text { cardioversion for patients } \\
\text { with unknown duration or } \\
\text { AF or with AF longer than } \\
48 \text { hours }\end{array}$ & $\begin{array}{l}\text { CHADS }_{2} \text { score } \geq 1 \text { or } \mathrm{CHADS}_{2} \text { score } 0 \\
\text { with persistent AF should receive warfarin } \\
\text { (documented INR 2-3) for } 3 \text { weeks pre- } \\
\text { ablation }\end{array}$ & $\begin{array}{l}\text { Recommend adherence to the } \mathrm{ACC} / \\
\text { AHA/ESC } 2006 \text { guidelines regarding } \\
\text { anticoagulation for cardioversion }\end{array}$ \\
\hline & & $\begin{array}{l}\text { CHADS } 2 \text { score } 0 \text { with paroxysmal AF may } \\
\text { receive either ASA } 75-325 \text { mg daily or } \\
\text { warfarin Recommend bridging with IV } \\
\text { UFH or SC LMWH (doses not specified) }\end{array}$ & $\begin{array}{l}\text { "Some Task Force members" recommend } \\
\text { bridging with enoxaparin } 0.5-1 \mathrm{mg} / \mathrm{kg} \\
\text { BID until the evening prior to ablation }\end{array}$ \\
\hline \multirow[t]{3}{*}{$\begin{array}{l}\text { Intra-ablation } \\
\text { Recommendations }\end{array}$} & $\begin{array}{l}\text { "Higher intensity" } \\
\text { anticoagulation may reduce } \\
\text { intra-procedural } \\
\text { thromboembolism }\end{array}$ & $\begin{array}{l}\text { Immediately after left atrial access is } \\
\text { established give IV UFH bolus } 100-140 \\
\text { units } / \mathrm{kg} \text { followed by an infusion of } 15-18 \\
\text { units/kg/hour }\end{array}$ & $\begin{array}{l}\text { Prior to or immediately on transseptal } \\
\text { puncture give IV UFH bolus } 100 \mathrm{units} / \mathrm{kg} \\
\text { followed by an infusion of } 10 \mathrm{units} / \mathrm{kg} / \\
\text { hour }\end{array}$ \\
\hline & & $\begin{array}{l}\text { ACT target at least } 250 \mathrm{~s} \text { and up to } 350-400 \\
\mathrm{~s}\end{array}$ & $\begin{array}{l}\text { ACT target 300-350 s May target ACT } \\
350-400 \mathrm{~s} \\
\text { if } \\
\text { significant atrial enlargement or } \\
\text { spontaneous echo contrast is present }\end{array}$ \\
\hline & & $\begin{array}{l}\text { Discontinue UFH infusion after all } \\
\text { catheters have been removed from the left } \\
\text { atrium }\end{array}$ & $\begin{array}{l}\text { Discontinue UFH and remove sheaths } \\
\text { when ACT }<200 \text { s or reverse UFH with } \\
\text { protamine }\end{array}$ \\
\hline \multirow[t]{3}{*}{$\begin{array}{l}\text { Post-ablation } \\
\text { Recommendations }\end{array}$} & $\begin{array}{l}\text { No specific } \\
\text { recommendations regarding } \\
\text { AF ablation, but } \\
\text { recommend anticoagulation } \\
\text { for at least } 4 \text { weeks post- } \\
\text { cardioversion }\end{array}$ & $\begin{array}{l}\text { Re-start warfarin the evening of procedure } \\
\text { or the following morning in all patients }\end{array}$ & $\begin{array}{l}\text { Re-start anticoagulation within } 4-6 \text { hours } \\
\text { post-sheath removal }\end{array}$ \\
\hline & & $\begin{array}{l}\text { May use IV UFH or SC LMWH for } \\
\text { bridging until INR } \geq 2\end{array}$ & $\begin{array}{l}\text { Use "therapeutic loading doses" of IV } \\
\text { UFH or SC enoxaparin and continue until } \\
\text { therapeutic INR "Many of the members } \\
\text { of this Task Force empirically and } \\
\text { independently arrived at a dose of } \\
\text { enoxaparin } 0.5 \mathrm{mg} / \mathrm{kg} \text { BID since an } \\
\text { unacceptable incidence of bleeding } \\
\text { complications has been observed at a } \\
\text { dose of } 1 \mathrm{mg} / \mathrm{kg} \text { BID." }\end{array}$ \\
\hline & & Continue warfarin for at least 3-6 months & $\begin{array}{l}\text { Continue warfarin for at least } 2 \text { months } \\
\text { Determine duration of anticoagulation } \\
\text { with warfarin on the patient's risk factors } \\
\text { and not on the presence or type of AF }\end{array}$ \\
\hline
\end{tabular}




\begin{tabular}{|c|c|c|c|}
\hline & $\begin{array}{l}\text { ACC/AHA/ESC } \\
\text { Guidelines for the } \\
\text { Management of Patients } \\
\text { with Atrial } \\
\text { Fibrillation (2006) [1] }\end{array}$ & $\begin{array}{l}\text { Venice Chart International Consensus } \\
\text { Document on Atrial Fibrillation Ablation } \\
\text { (2007) [25] }\end{array}$ & $\begin{array}{l}\text { HRS/EHRA/ECAS Expert Consensus } \\
\text { Statement } \\
\text { on Catheter and Surgical Ablation of } \\
\text { Atrial } \\
\text { Fibrillation: Recommendations for } \\
\text { Personnel, } \\
\text { Policy, Procedures and Follow-Up } \\
(2007) \text { [22] }\end{array}$ \\
\hline & & $\begin{array}{l}\mathrm{CHADS}_{2} \text { score } \geq 2 \text { suggest continue long- } \\
\text { term warfarin (INR 2-3). May consider } \\
\text { discontinuing warfarin in select patients } \\
\text { without evidence of recurrent AF. If } \\
\text { warfarin discontinued, recommend ASA } \\
75-325 \text { mg daily unless contraindicated. } \\
\text { For others without evidence of recurrent AF } \\
\text { consider: CHADS } \\
\text { warfarin } \mathrm{CHADS}_{2} \text { score } 1=\text { ASA or } \\
\text { wano treatment }\end{array}$ & $\begin{array}{l}\mathrm{CHADS}_{2} \text { score } \geq 2 \text { continue warfarin } \\
\text { indefinitely }\end{array}$ \\
\hline Other & $\begin{array}{l}\text { "The long-term efficacy of } \\
\text { catheter ablation to prevent } \\
\text { recurrent AF requires } \\
\text { further study...it remains } \\
\text { uncertain whether apparent } \\
\text { cures represent elimination } \\
\text { of AF or transformation } \\
\text { into an asymptomatic orm } \\
\text { of paroxysmal AF. The } \\
\text { distinction has important } \\
\text { implications for the } \\
\text { duration of anticoagulation } \\
\text { therapy in patients with risk } \\
\text { factors for stroke associated } \\
\text { with AF." }\end{array}$ & $\begin{array}{l}\text { "An overlying concern is the absence of } \\
\text { long-term data in large numbers of patients } \\
\text { following catheter ablation of AF. We need } \\
\text { a prospective study not only on the } \\
\text { incidence of recurrence of AF beyond the } \\
\text { 'blanking period,' but also on the incidence } \\
\text { of stroke in the absence of anticoagulation } \\
\text { therapy, especially in patients with risk } \\
\text { factors for stroke. Data from relatively } \\
\text { small studies suggest that the stroke } \\
\text { incidence is low, but the incidence of AF } \\
\text { recurrence, both manifest and } \\
\text { asymptomatic, is uncertain. Future well- } \\
\text { conducted randomized trials have to } \\
\text { establish the best anticoagulation protocol } \\
\text { after ablation of AF, according to the } \\
\text { clinical characteristics of the patients, } \\
\text { especially their thromboembolic risk." }\end{array}$ & $\begin{array}{l}\text { "...the Task Force recognizes that } \\
\text { patients who have undergone catheter } \\
\text { ablation of AF represent } \\
\text { a new and previously unstudied } \\
\text { population of patients. Clinical trials are } \\
\text { therefore needed to define the stroke risk } \\
\text { of this patient population and to } \\
\text { determine whether the risk factors } \\
\text { identified in CHADS or other scoring } \\
\text { systems apply to these patients." }\end{array}$ \\
\hline
\end{tabular}

$\mathrm{ACC}=\mathrm{American}$ College of Cardiology; $\mathrm{ACT}=$ activated clotting time; $\mathrm{AF}=$ atrial fibrillation; $\mathrm{AHA}=\mathrm{American}$ Heart Association, $\mathrm{ASA}=\mathrm{aspirin}$; $\mathrm{BID}=$ twice daily; $\mathrm{CAD}=$ coronary artery disease; $\mathrm{DM}=$ diabetes mellitus; $\mathrm{ECAS}=$ European Cardiac Arrhythmia Society; EHRA=European Heart Rhythm Association; ESC=European Society of Cardiology; HF=heart failure; HRS=Heart Rhythm Society; HTN=hypertension;

INR=international normalized ratio; IV UFH=intravenous unfractionated heparin; LVEF=left ventricular ejection fraction; s=seconds; SC LMWH=subcutaneous low molecular weight heparin; TIA=transient ischemic attack.

${ }^{a}$ Less validated or weaker risk factors: female gender, age 65-74, CAD, thyrotoxicosis; Moderate risk factors: age $\geq 75, \mathrm{HTN}, \mathrm{HF}, \mathrm{LVEF} \leq 35 \%$, DM; High risk factors: previous stroke, TIA, or embolism, mitral stenosis, prosthetic heart valve. 